\title{
Simultaneous Vehicle Routing and Charging Station Siting for Commercial Electric Vehicles
}

\author{
Owen Worley, Diego Klabjan, and Timothy M. Sweda \\ Department of Industrial Engineering and Management Sciences \\ Northwestern University \\ Evanston, Illinois 60208 \\ Email: owen.worley@u.northwestern.edu; d-klabjan@northwestern.edu; tsweda@u.northwestern.edu
}

\section{INTRODUCTION}

As organizations electrify their vehicle fleets in order to lower costs, reduce emissions, and improve their public image, they face the problem of providing charging access to their vehicles on the road. Electric vehicles (EVs) have a much shorter driving range than comparable gasoline-powered vehicles, and without charging capabilities away from the depot, an EV's route length is constrained by its battery capacity. This is especially problematic if customers are located far from the depot, since it may not be possible to visit a customer and return to the depot on a single charge. Because public charging access is not usually available for EVs, the fleet owner may need to install or rent charging stations away from the depot for the EVs to use.

In this paper, we formulate the problem of locating charging stations and also designing EV routes as a discrete integer programming optimization problem, based on the classic Vehicle Routing Problem (VRP).

\section{LITERATURE REVIEW}

Previous approaches to locating refueling stations usually involve a variation of the set covering model, where vehicle flows along arcs are known and the objective is to locate refueling stations that cover all vehicle flows such that the total cost is minimized. In [1], [2], [3] the flow refueling location model is used, which locates a fixed number of stations to maximize the refueling of deterministic origin-destination flows. However, the optimal solution may not cover all vehicle flows. An alternative method is proposed in [4], which heuristically solves the arc demand coverage problem to locate compressed natural gas $(\mathrm{CNG})$ refueling stations when vehicle flows are given. The optimal solution from this approach is guaranteed to cover all vehicle flows, but the assumption that vehicle flows are known a priori is difficult to justify for alternative fuels (such as CNG) that are still in development. In [5], an agent-based modeling approach is employed for locating hydrogen refueling infrastructure. The simulation incorporates randomized vehicle flows, which are influenced over time as hydrogen refueling stations are located and may also increase or decrease based on adoption rates of vehicles fueled by hydrogen. Driver agents are modeled separately from investor agents (who install the infrastructure), so the agent behaviors are reactive rather than coordinative.

We differ from all these in that we consider the routing and recharging/refueling locating problems simultaneously and directly. In contrast to the agent based approach discussed above, the routing and locating are performed in coordination, so a true optimal solution will be found rather than, potentially, a local Pareto optimal solution as in the agent based approach.

\section{MODEL}

The integer programming model finds an optimal (least cost) set of routes and charging station locations, where vehicles originating from a single depot must satisfy all demand and cannot travel a distance greater than their range without visiting a charging station. The objective is to minimize the sum of the travel costs, recharging costs, and costs of locating charging stations. Other than the charging aspects, this setting is identical to the traditional VRP assumptions.

We let $N$ denote the set of customer nodes, $C$ denote the set of potential charging station sites, $O$ denote the depot, $V=N \cup C \cup\{O\}$ denote the set of all nodes in the network, $A$ denote the set of all arcs, and $S$ denote the set of vehicles. We also let $K$ denote the set of all route parts, where a part is defined as a path that begins at the depot or a charging station node, ends at the depot or a charging station node, does not include any other charging stations or the depot in between, and has total length less than or equal to the range of the vehicle. The decision variables include $x_{i j k s}$, which equals 1 if part $k$ is traversed by vehicle $s$ and includes arc $(i, j)$ and 0 otherwise; $y_{i k s}$, which equals 1 if part $k$ is traversed by vehicle $s$ and includes node $i$ and 0 otherwise; and $z_{i}$, which equals 1 if a charging station is opened/deployed at node $i$ and 0 otherwise. 
The parameters include $c_{i j}$, the cost of traversing arc $(i, j) \in A ; p_{i}$, the cost of recharging at node $i \in C ; h_{i}$, the cost of opening a charging station at node $i \in C$; $q_{i}$, the demand of customer $i \in N ; Q_{s}$, the maximum capacity of vehicle $s \in S$; and $L$, the maximum distance a vehicle can travel between recharges (assumed to be the same for all vehicles). In addition, let $M$ be a large number.

A. The integer programming formulation reads as follows:

$$
\begin{aligned}
& \min \sum_{(i, j) \in A} \sum_{k \in K} \sum_{s \in S} c_{i j} x_{i j k s}+ \\
& \sum_{i \in C} \sum_{k \in K} \sum_{s \in S} p_{i} y_{i k s}+\sum_{i \in C} h_{i} z_{i} \\
& \text { s.t. } \sum_{k \in K} \sum_{s \in S} y_{i k s}=1 \quad \forall i \in N \\
& \sum_{i \in N} \sum_{k \in K} q_{i} y_{i k s} \leq Q_{s} \quad \forall s \in S \\
& \sum_{i \in V} \sum_{k \in K} x_{i j k s}-\sum_{m \in V} \sum_{k \in K} x_{j m k s}=0 \\
& \forall j \in C \cup\{O\}, s \in S \\
& \sum_{i \in V} x_{i j k s}-\sum_{m \in V} x_{j m k s}=0 \\
& \forall j \in N, k \in K, s \in S \\
& \sum_{j \in V} x_{i j k s} \geq y_{i k s} \forall i \in N, k \in K, s \in S \\
& \sum_{j \in V} x_{i j k s} \leq M y_{i k s} \\
& \forall i \in C, k \in K, s \in S \\
& \sum_{k \in K} \sum_{s \in S} y_{i k s} \leq M z_{i} \quad \forall i \in C \\
& \sum_{i \in V} \sum_{j \in V} c_{i j} x_{i j k s} \leq L \quad \forall k \in K, s \in S \\
& M * \sum_{i \in V \backslash R} \sum_{j \in R} \sum_{k \in K} x_{i j k s} \geq \sum_{j \in R} \sum_{k \in K} y_{i k s} \\
& \forall R \subseteq V \backslash\{O\}, s \in S \\
& M * \sum_{i \in V \backslash R} \sum_{j \in R} x_{i j k s} \geq \sum_{j \in R} y_{i k s} \\
& \forall R \subseteq V \backslash\{O\}, s \in S, k \in K \\
& x_{i j k s} \in\{0,1\} \quad \forall(i, j) \in A, k \in K, s \in S \\
& y_{i k s} \in\{0,1\} \quad \forall i \in V, k \in K, s \in S \\
& z_{i} \in\{0,1\} \quad \forall i \in C
\end{aligned}
$$

The objective function minimizes the sum of the total transportation, recharging, and charging station placement costs. Constraints (1) require that every customer be visited, while constraints (2) enforce the vehicle capacities. Constraints (3) are the flow balance constraints for vehicles at charging station nodes, and constraints (4) are the flow balance constraints for route parts at customer nodes. Constraints (5) link the arc and node variables at customer nodes, and constraints (6) link the arc and node variables at charging station nodes. Constraints (7) ensure that vehicles only stop to recharge at nodes where charging stations have been placed. Constraints (8) enforce range constraints on the vehicles (i.e., how far a vehicle can travel until it must recharge), and constraints (9) and (10) are the subtour elimination constraints.

\section{Case Study}

We will demonstrate the model using pickup/delivery demand data from a large express parcel delivery company, together with vehicle range and power consumption typical of the electric light trucks on the market, typical electricity rates in the Chicago area, and an estimate of charging station siting costs based on the cost of equipment plus an additional fee for rental of the location itself.

Fig. 1 portrays a small example of a generated solution to the model: there are two routes, labelled '1' and '2'; all nodes other than the depot (the central node) are both demand nodes and potential charging station locations; and the nodes that were chosen by the model to be charging station locations are highlighted with a box (in the model, the depot is always a charging station). Note that optimal routes are not always polygons, as in the classical VRP or TSP (traveling salesman problem); charging can cause routes to be pinched off or doubled back on themselves.

The model also allows us to discern the benefits of field charging. For example, for the instance whose solution is shown in Fig. 1, when we disallow field charging, an additional vehicle must be used, and the model cost (not including the cost of the additional vehicle) rises by $21 \%$.

\section{FUTURE WORK}

We plan to perform more extensive tests of the model, in a variety of realistic instances for pickup/delivery and perhaps other applications. We will also develop solution methodologies for large instances, which are currently challenging to compute. We feel that the relevance of 


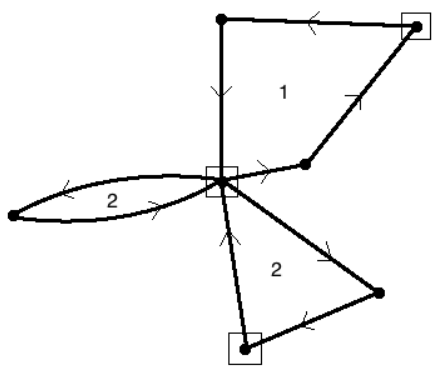

Fig. 1. Example model output. (Distances not to scale.)

the model for EV fleets, and the clear differences of our initial case study results from the non-charging VRP results, provide ample reason to move forward.

\section{REFERENCES}

[1] Kuby, M., \& Lim, S. (2005). The flow-refueling location problem for alternative-fuel vehicles. Socio-Economic Planning Sciences, $39,125-145$

[2] Kuby, M., \& Lim, S. (2007). Location of Alternative-Fuel Stations Using the Flow-Refueling Location Model and Dispersion of Candidate Sites on Arcs. Networks and Spatial Economics, 7, 129152.

[3] Upchurch, C., Kuby, M., \& Lim, S. (2009). A Model for Location of Capacitated Alternative-Fuel Stations. Geographical Analysis, 41, 85-106.

[4] Boostani, A., Ghodsi, R., \& Miab, A. K. (2010). Optimal Location of Compressed Natural Gas (CNG) Refueling Station Using the Arc Demand Coverage Model. 2010 Fourth Asia International Conference on Mathematical/Analytical Modelling and Computer Simulation, Kota Kinabalu, Malaysia (pp. 193-198).

[5] Stephan, C., \& Sullivan, J. (2004). Growth of a Hydrogen Transportation Infrastructure. Agent 2004 - Social Dynamics: Interaction, Reflexivity and Emergence, Chicago, IL. 